Classification

Physics Abstracts

$06.20-06.46-06.94-22.48-97.82$

\title{
Principes de la thermographie microonde par corrélation
}

\author{
A. Mamouni, Y. Leroy, J. C. Van de Velde et L. Bellarbi \\ Centre Hyperfréquences et Semiconducteurs $\left({ }^{*}\right)$, \\ Université de Lille-I, Bât. P4, 59655 Villeneuve d'Ascq Cedex, France
}

(Reçu le 18 juillet 1983, révisé le 7 novembre, accepté le 10 novembre 1983)

\begin{abstract}
Résumé. - La Thermographie Microonde par Corrélation (T.M.O.C.) est un cas particulier de la Thermographie Microonde (T.M.O.) - ou radiométrie appliquée à la mesure du bruit thermique - qui intéresse actuellement les applications médicales (méthodes diagnostiques et d'investigations fondées sur la mise en évidence des gradients thermiques existant dans les tissus vivants). A la différence des méthodes classiques de T.M.O., la T.M.O.C. met en œuvre une détection cohérente du bruit. Nous présentons les principes de cette méthode et analysons certaines de ses caractéristiques. La partie expérimentale de cette étude est effectuée à l'aide d'un système construit au laboratoire fonctionnant en bande $\mathrm{S}(2-4 \mathrm{GHz})$.
\end{abstract}

\begin{abstract}
Correlation Microwave Thermography (CMWT) is a particular case of Microwave Thermography (MWT) or radiometry applied to the measurement of thermal noise. It concerns at present biomedical engineering (diagnostic methods based on the investigation into thermal gradients which occur in living tissues). This paper presents the basis of this method and analyses some of its characteristics. The experimental part of this study is carried out using a system built in our laboratory, working in the $\mathrm{S}$ band $(2-4 \mathrm{GHz})$.
\end{abstract}

\section{Introduction.}

La Thermographie Microonde (T.M.O.) consiste à déterminer la température de milieux dissipatifs à partir de la mesure des signaux de bruit thermiques émis par ces milieux.

Des applications développées, au cours de ces dernières années, concernent la médecine :

- méthodes diagnostiques mettant en évidence l'existence de gradients thermiques qui ont généralement des causes pathologiques (cancers du sein, du cerveau, pathologies ostéoarticulaires, rhumatismes) [1] à [8] ;

- contrôle atraumatique de la température au cours d'un traitement fondé sur le chauffage des tissus (thérapeutique du cancer par hyperthermie) $[9,10]$.

Les systèmes utilisés actuellement sont fondés sur la transposition aux applications médicales des techniques éprouvées couramment utilisées en radioastronomie (radiomètre de Dicke) [11]. Schématiquement, on peut dire que ces systèmes sont constitués d'une sonde, destinée à être appliquée sur la zone de tissus sous investigation, reliée à un récepteur de grande sensibilité appelé radiomètre. Des travaux

(*) L.A. au CNRS No 287.

REVUe DE PHYSIQUe APPLIQUÉE. - T. 19, № 3, MARS 1984 actuellement en cours ont pour but de définir des algorithmes de Reconnaissance d'Objets Thermiques permettant d'exploiter les signaux radiométriques pour en déduire la répartition de température régnant dans les tissus [12] à [16].

Parallèlement, nous essayons aussi de transposer au cas des applications médicales les perfectionnements que les radioastronomes ont apportés à leurs techniques.

Le procédé de ce type que nous examinons ici est déduit de l'interféromètre à commutation de phase proposé par Ryle [17] pour l'observation de sources stellaires de faible intensité. Il conduit à utiliser deux sondes qui sont reliées à un corrélateur suivi d'un récepteur radiométrique. La transposition de ce procédé aux cas d'expériences de radiométrie effectuées à courte distance semble intéressante pour les raisons suivantes.

Dans les systèmes de première génération qui n'utilisent qu'une seule sonde, le signal radiométrique est dû à l'émission thermique de la totalité du milieu dissipatif couplé à la sonde.

Dans le cas envisagé ici que nous avons appelé Thermographie Microonde par Corrélation (T.M.O.C.), seul le bruit thermique émis dans la partie commune aux volumes couplés aux deux sondes contribue au 
signal de sortie du récepteur (Fig. 1). Cette caractéristique est intéressante [18-20] car :

- le nouveau volume de couplage est généralement plus petit que le précédent ; on peut s'attendre à ce que la nouvelle méthode conduise à une meilleure résolution spatiale;

- il est possible d'agir sur la position relative des sondes, d'où une souplesse supplémentaire du point de vue expérimental;

- il est également possible d'introduire un déphasage supplémentaire dans l'un des bras du corrélateur, et par conséquent d'utiliser cette possibilité pour augmenter la richesse des informations obtenues.

Les points développés dans cet article concernent :

- les principes de la T.M.O.C.;

- la construction d'un dispositif expérimental fonctionnant en bande $\mathrm{S}(2-4 \mathrm{GHz})$;

- les premières études effectuées sur la contribution d'un volume élémentaire faisant partie d'un milieu dissipatif (eau).

\section{Principe de la thermographie microonde par cor- rélation (T.M.O.C.).}

Le système de T.M.O.C. (Fig. 2) comporte deux sondes reliées à un additionneur de puissance, par l'intermédiaire d'un modulateur de phase $\left(0^{\circ}-180^{\circ}\right)$ commandé alternativement par un générateur d'impulsions (rapport cyclique $\delta=0,5$ ), et par un déphaseur introduisant une phase $\Delta \Phi=2 \Pi \Delta l / \lambda$ ( $\lambda$ étant la longueur d'onde considérée, une différence de marche supplémentaire $\Delta l$ est créée dans le bras considéré).

Initialement les longueurs électriques des deux bras sont identiques $(\Delta l=0)$. L'additionneur de puissance est suivi d'un récepteur faible bruit, d'un détecteur

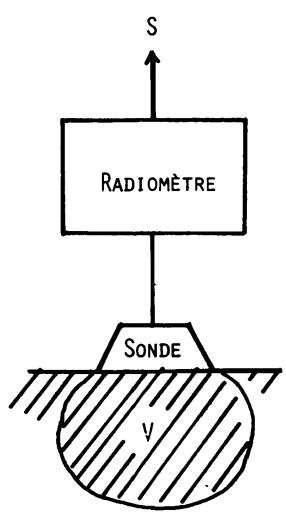

T.M.O.

PREMIËRE GÉNERATION

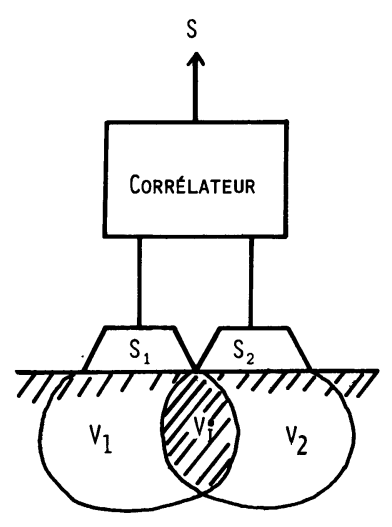

T.M.O.C,
Fig. 1. - Schéma de principe des systèmes T.M.O. et T.M.O.C. En hachuré les zones de milieu dissipatif dont l'émission thermique contribue au signal radiométrique $S$.

[Principle of the systems MWT and CMWT (T.M.O. and T.M.O.C. in frensh) (the hatched areas $V$ and $V_{i}$ contribute to the radiometric signals.).]

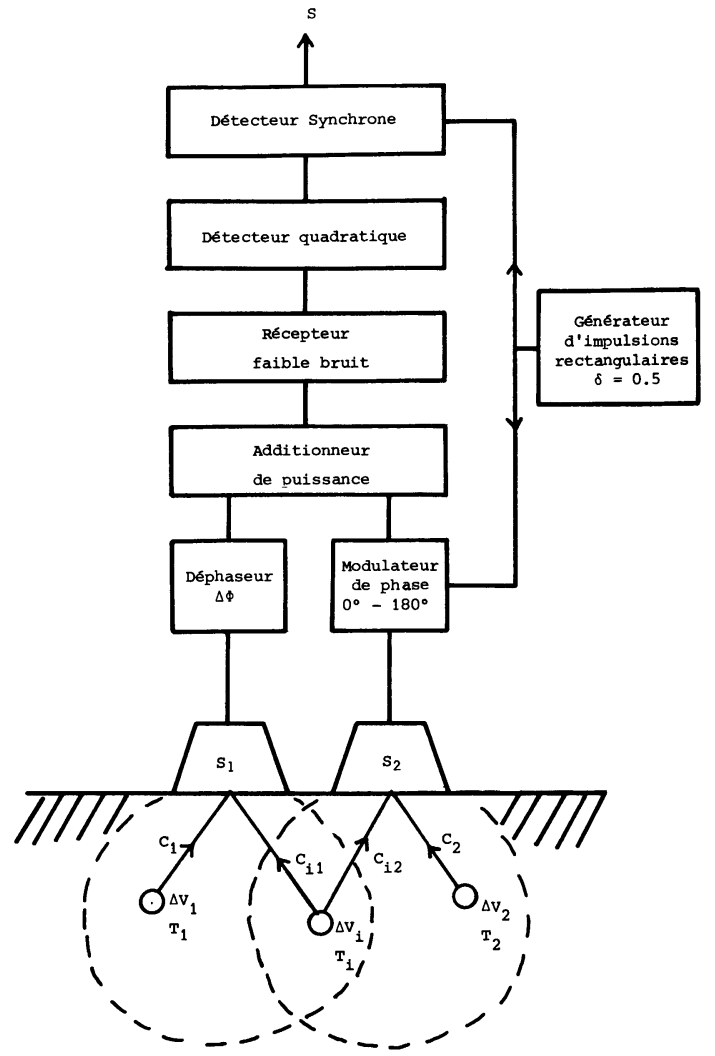

Fig. 2. - Schéma de principe d'un Thermographe Microonde par Corrélation.

[Synoptic of a system of Correlation Microwave Thermography.]

quadratique, d'une détection synchrone, ou d'un système de filtrage numérique.

Chaque molécule du milieu dissipatif émet un signal de bruit thermique; tout volume élémentaire $\Delta v_{i}$ du milieu constitué d'un grand nombre de molécules se comporte également comme un émetteur; son diagramme de rayonnement est isotrope.

La puissance émise par chaque volume est proportionnelle à sa température absolue (loi de RayleighJeans). Si l'on considère une bande passante étroite, le couplage entre un volume élémentaire $\Delta v_{i}$ et une sonde est caractérisé par une grandeur complexe :

$$
c_{i}=C_{i} \Phi_{i} .
$$

Des études antérieures sur le rayonnement thermique nous ont montré que par application du théorème de réciprocité, les paramètres $C_{i}$ peuvent être déduits de la connaissance du champ rayonné dans chaque volume élémentaire lorsque la même sonde émet une onde monochromatique dans le même milieu dissipatif. Le fait d'utiliser ainsi un signal auxiliaire a été appelé Mode Actif [12].

On appelle $C_{i 1}, C_{i 2}, C_{1}$ et $C_{2}$ les paramètres de couplage entre les sondes $\mathrm{S}_{1}$ et $\mathrm{S}_{2}$ et les volumes $\Delta V_{i}$, $\Delta V_{1}$ et $\Delta V_{2} . \Delta V_{1}$ est couplé à $S_{1}, \Delta V_{2}$ à $S_{2} ;$ mais $\Delta V_{i}$ est couplé à la fois à $S_{1}$ et $S_{2}$. 


$$
\begin{aligned}
& c_{i 1}=C_{i 1}\left\lfloor\Phi_{i 1}\right. \\
& c_{i 2}=c_{i 2}\left\lfloor\Phi_{i 2}\right. \\
& c_{1}=C_{1} \Phi_{1} \\
& c_{2}=C_{2} \Phi_{2} .
\end{aligned}
$$

Evaluons les contributions au signal de sortie $\mathrm{S}$ des signaux thermiques générés par $\Delta V_{1}, \Delta V_{2}$ et $\Delta V_{i}$, portés respectivement aux températures $T_{1}, T_{2}$ et $T_{i}$. Lorsque le modulateur de phase est en position $0^{\circ}$, cette contribution est proportionnelle à

$$
\begin{aligned}
S_{1}= & T_{1} C_{1}^{2}+T_{2} C_{2}^{2}+T_{i}\left[C_{i 1}^{2}+C_{i 2}^{2}\right. \\
& \left.+2 C_{i 1} C_{i 2} \cos \Psi \cdot \cos \left(\Phi_{i 1}-\Phi_{i 2}+\Delta \Phi\right)\right] .
\end{aligned}
$$

Lorsque le modulateur est en position $180^{\circ}$,

$$
\begin{aligned}
S_{2}= & T_{1} C_{1}^{2}+T_{2} C_{2}^{2}+T_{i}\left[C_{i 1}^{2}+C_{i 2}^{2}\right. \\
& \left.-2 C_{i 1} C_{i 2} \cos \Psi \cdot \cos \left(\Phi_{i 1}-\Phi_{i 2}+\Delta \Phi\right)\right] .
\end{aligned}
$$

Dans ces expressions, les doubles produits correspondent aux signaux reçus par $S_{1}$ et $S_{2}$ qui sont corrélés parce qu'ils proviennent des mêmes émetteurs de bruit thermique. Le terme $\cos \Psi$ exprime que les sondes $\mathrm{S}_{1}$ et $\mathrm{S}_{2}$ sont sensibles à des rayonnements pour lesquels les directions de polarisation du champ électrique peuvent être différentes et font entre elles un angle $\Psi$ (Annexe I).

Les volumes précités $\Delta V_{i}, \Delta V_{1}$ et $\Delta V_{2}$ produisent donc en sortie un signal moyen dont l'amplitude est proportionnelle à

$$
T_{i} C_{i 1} C_{i 2} \cos \Psi \cdot \cos \left(\Phi_{i 1}-\Phi_{i 2}+\Delta \Phi\right) .
$$

Par conséquent, seule l'émission thermique de la partie $V_{i}$ commune aux deux volumes de couplage $V_{1}$ et $V_{2}$ des deux sondes contribue au signal de sortie $\mathrm{S}$. A une constante près, la contribution totale de $V_{i}$ correspond à la somme de termes tels ceux qui sont exprimés par la relation (8). La méthode proposée est donc insensible à l'émission thermique de volumes couplés uniquement à l'une des sondes. Ces caractéristiques constituent un élément nouveau en radiométrie.

\section{Construction d'un corrélateur fonctionnant en ban- de $\mathbf{S}$.}

Le schéma de principe du montage que nous avons d'abord construit est donné figure 3. Dans ce système on reconnaît le corrélateur - à droite du plan $\mathrm{AB}$ que l'on a connecté à une source de bruit par l'intermédiaire d'un coupleur $180^{\circ}$. Ce dispositif permet de définir les tests et les mises au point qui sont effectués expérimentalement,

Estimons la puissance de bruit captée par le récepteur, schématisé par la résistance $R_{3}$ (toutes les résistances du circuit sont des charges adaptées). Les signaux correspondants proviennent de la source de bruit blanc (température $T$ ) et de $R^{\prime}$ (température

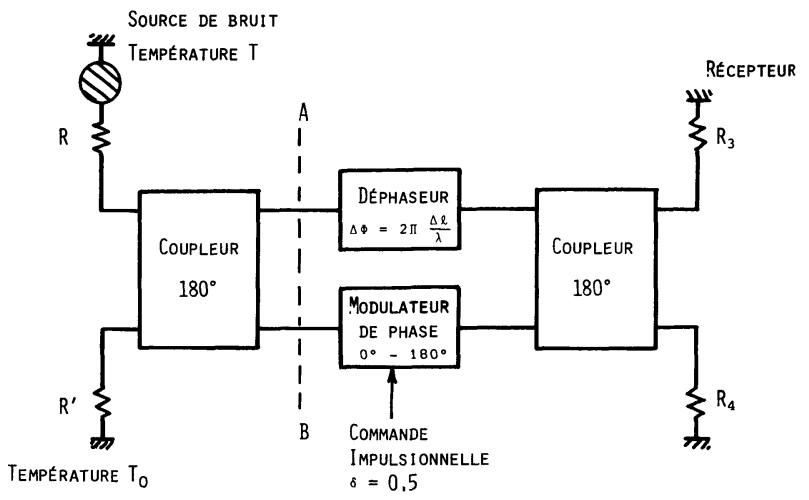

Fig. 3. - Montage considéré pour les tests du corrélateur.

[Set-up used in order to test the correlator.]

ambiante $T_{0}$ ). La bande passante $\Delta f$ que nous considérons d'abord est très réduite; ainsi, les expressions instantanées de ces signaux $v_{1}(t)$ et $v_{2}(t)$ générées par $R$ et $R^{\prime}$ sont assimilables à celles de composantes monochromatiques $V_{1} \underline{L}^{\circ}$ et $V_{2} L^{\circ}$; ces signaux sont évidemment décorrélés.

Lorsque le modulateur de phase est en position $0^{\circ}$, on fournit au récepteur une tension :

$$
\begin{aligned}
v_{1}^{\prime \prime}(t)=\frac{1}{2}\left[V_{1} \varphi\right. & \left.+\Delta \Phi+V_{1} \varphi\right]+ \\
& +\frac{1}{2}\left[V_{2} \varphi+\Pi+V_{2} \varphi+\Delta \Phi\right]
\end{aligned}
$$

avec $\varphi$, le déphasage introduit par la propagation des signaux dans le circuit. La puissance correspondante est :

$P_{1}=\frac{1}{2 R}\left[V_{1}^{2} \operatorname{eff}(1+\cos \Delta \Phi)+V_{2}^{2} \operatorname{eff}(1-\cos \Delta \Phi)\right]$

soit, par application du théorème de Nyquist

$$
P_{1}=k\left[T_{0}+\frac{1}{2}\left(T-T_{0}\right)(1+\cos \Delta \Phi)\right] \Delta f .
$$

Lorsque le modulateur de phase est en position $180^{\circ}$, on fournit au récepteur une tension

$$
\begin{aligned}
v_{2}^{\prime \prime}(t)=\frac{1}{2}\left[V_{1} \varphi+\Delta \Phi\right. & \left.+V_{1} \varphi+\Pi\right]+ \\
& +\frac{1}{2}\left[V_{2} \varphi+\Delta \Phi+V_{2} \varphi\right]
\end{aligned}
$$

soit une puissance

$$
\begin{aligned}
& P_{2}=\frac{1}{2 R}\left[V_{1}^{2} \operatorname{eff}(1-\cos \Delta \Phi)+V_{2}^{2} \operatorname{eff}(1+\cos \Delta \Phi)\right] \\
& P_{2}=k\left[T_{0}+\frac{1}{2}\left(T-T_{0}\right)(1-\cos \Delta \Phi)\right] \Delta f
\end{aligned}
$$

$\mathrm{Au}$ cours d'une période de modulation, le signal détecté vaut alternativement $P_{1}$ et $P_{2}$; pour un hertz de bande passante, son amplitude crête à crête a pour expression :

$$
P_{\mathrm{S}}=k\left(T-T_{0}\right) \cos \Delta \Phi .
$$


D'après les relations (10) et (13), on voit d'une part que l'atténuation, ou l'amplification, que l'on introduisait dans l'un des bras du système modifie simplement la relation (15) par mise en facteur de la quantité correspondante. D'autre part, si la modulation de phase est entachée d'erreurs (erreurs $\Delta \Phi_{\mathrm{c}}$ en position $180^{\circ}$, et $\Delta \Phi_{0}$ en position $0^{\circ}$ ) la relation (15) devient :

$$
P_{\mathrm{S}}=\frac{1}{2} k\left(T-T_{0}\right)\left[\cos \left(\Delta \Phi-\Delta \Phi_{\mathrm{c}}\right)+\cos \left(\Delta \Phi-\Delta \Phi_{0}\right)\right] .
$$

La relation (16) indique qu'il est indispensable de minimiser les erreurs $\Delta \Phi_{\mathrm{c}}$ et $\Delta \Phi_{0}$. Après plusieurs essais, nous avons choisi de réaliser le modulateur de phase à l'aide d'un circulateur et d'un commutateur relié soit à un court circuit, soit à un circuit ouvert (Fig. 4). Le déphaseur $(\Delta \Phi)$ est constitué d'un circulateur connecté à une ligne court circuitée de longueur variable. Nous avons vérifié que, aux fréquences où nous opérons (2-4 GHz) les quantités $\Delta \Phi_{\mathrm{c}}$ et $\Delta \Phi_{0}$ ne dépassent pas $2^{\circ}$.

Le récepteur est constitué d'un préamplificateur faible bruit à transistors à effet de champ (FET AsGa) (gain $30 \mathrm{~dB}$ - facteur de bruit $3 \mathrm{~dB}$ - bande de fréquences $2-4 \mathrm{GHz}$ ) suivi d'un récepteur hétérodyne avec amplificateur F.I. (5-500 MHz), d'une détection quadratique et d'un système de filtrage numérique. Ce récepteur a une sensibilité de l'ordre de $0,1^{\circ} \mathrm{C}$ pour un temps de mesure de $1 \mathrm{~s}$.

Le test de bon fonctionnement du corrélateur s'effectue à partir de l'analyse du signal de sortie enregistré en fonction de $\Delta l$. Cet interférogramme (Fig. 5) est en très bon accord avec son expression théorique déduite de (15)

$$
P_{\mathrm{S}}(\Delta l)=k\left(T-T_{0}\right) \int_{f_{1}}^{f_{2}} G(f) \cos \frac{2 \Pi f \Delta l}{C} \mathrm{~d} f
$$

avec $G(f)$ la réponse spectrale du récepteur relevée expérimentalement (Fig. 6).

Notons que l'on pourrait utiliser l'interférogramme $P_{\mathrm{S}}(\Delta l)$ pour en déduire $G(f)$ par transformée de Fourier (ceci est une méthode classique en spectroscopie).

Remarquons aussi que lorsque $G(f)$ est et $f_{2}$;

- constant pour les fréquences comprises entre $f_{1}$

- nul pour les autres fréquences;

la relation (17) s'écrit :

$$
P_{\mathrm{s}}(\Delta l) \doteq K\left(T-T_{0}\right)\left(f_{2}-f_{1}\right) \frac{\sin 2 \Pi \frac{\Delta l\left(f_{2}-f_{1}\right)}{c}}{2 \Pi \frac{\Delta l\left(f_{2}-f_{1}\right)}{c}} \cdot \cos \frac{2 \Pi\left(f_{1}+f_{2}\right) \Delta l}{c} .
$$

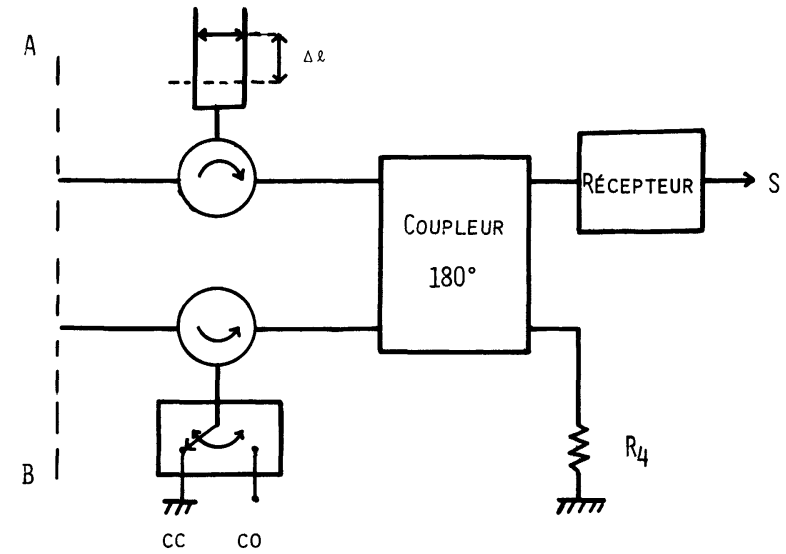

Fig. 4. - Réalisation pratique du corrélateur.

[Practical construction of the correlator.]

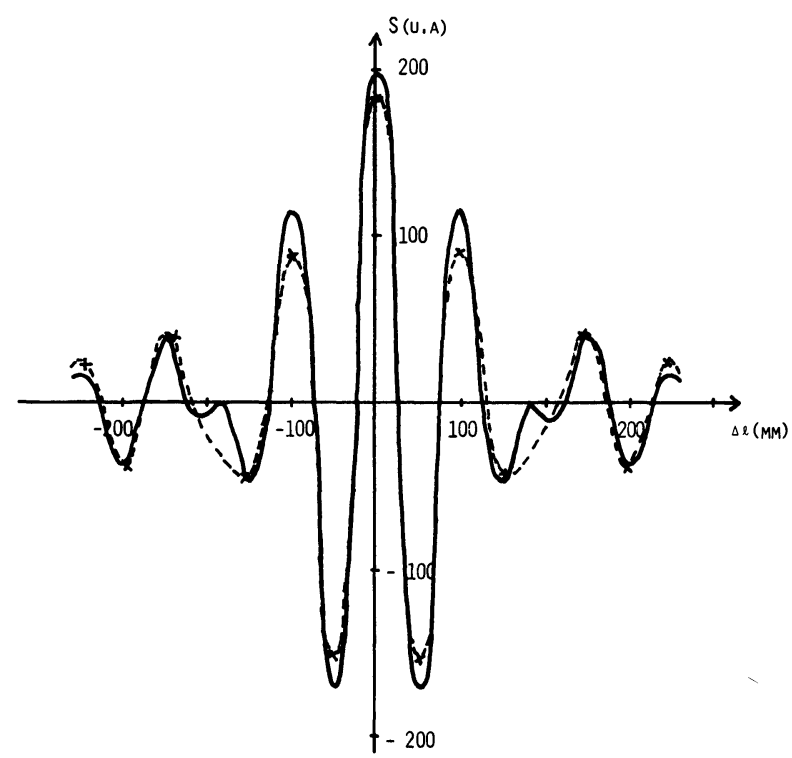

Fig. 5. - Interférogramme $S(\Delta l)$ obtenu à partir du cor- - - Valeurs calculées d'après (17) avec $G(f)$ donnée figure 6.

[Interferogram $S(\Delta l)$ obtained with the correlator experiment; -------- deduced from relation $(17),(G(f)$ is given in Fig. 6).] rélateur du type donné figure 3. - Enregistrement;
Cette approximation assez grossière nous permet cependant d'interpréter l'interférogramme de la 


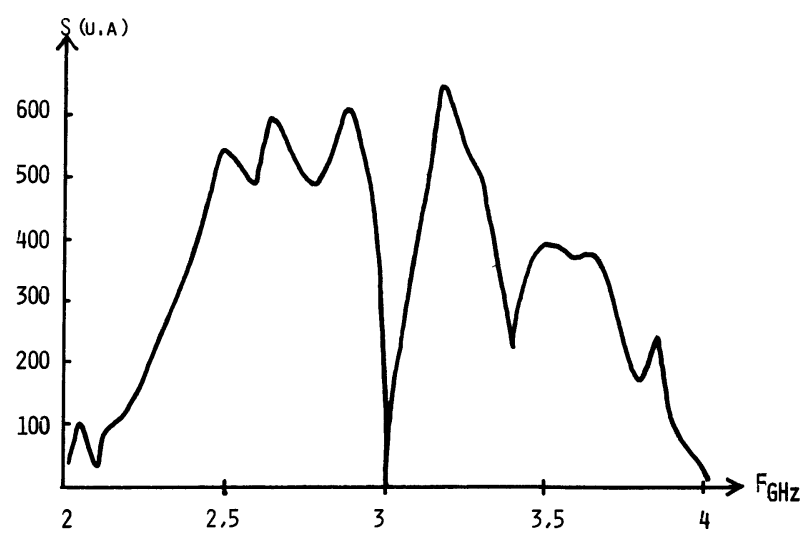

Fig. 6. - Réponse spectrale du récepteur relevée expérimentalement.

[Experimental spectral response of the receiver.]

$3 \mathrm{GHz}$ ) ; la distance entre les premiers zéros de l'enveloppe est voisine de $\frac{2 c}{f_{2}-f_{1}}\left(f_{1} \# 2.2 \mathrm{GHz}\right.$ et $f_{2} \# 3.8 \mathrm{GHz}$ ).

\section{Etude du signal détecté lorsque les sondes sont appliquées sur un milieu dissipatif à température uniforme.}

Le schéma du dispositif est donné figure 7. Nous conservons les éléments de la figure 3 situés à droite du plan $A B$ en y ajoutant les deux sondes $S_{1}$ et $S_{2}$. Actuellement ces sondes sont constituées par des tronçons de guide, remplis d'un diélectrique à faibles pertes, reliés au circuit par une transition guidecoaxial [12].

Le signal mesuré par le récepteur provient de la contribution des signaux thermiques émis à la fois :

- par le milieu dissipatif puis captés par les sondes;

- par les résistances $R_{3}$ et $R_{4}$, transmis par le circuit et réfléchis éventuellement par les interfaces sonde-milieu dissipatif (coefficient de réflexion $\rho$ ).

4.1 LES SONDES SONT SUPPOSÉES ADAPTÉES AU MILIEU DISSIPATIF $(\rho=0)$. - Dans ce cas, le signal détecté est nul : la thermographie microonde par corrélation possède la propriété d'être insensible aux signaux thermiques émis par un milieu porté à une température uniforme. Cette propriété se démontre par application du deuxième principe de la thermodynamique au système de la figure 7 considéré isotherme. Dans ce cas, le bilan des transferts radiatifs doit être en équilibre : les puissances de bruit thermiques cédées par les résistances $R_{3}$ et $R_{4}$ au milieu dissipatif doivent égaler les puissances cédées par le milieu aux mêmes résistances $R_{3}$ et $R_{4}$.

Cet équilibre est conservé quelles que soient les valeurs de $\Delta \Phi$ ou de la phase réalisée par le modulateur. En particulier, la puissance reçue par le récepteur ne dépend en aucune façon de la position relative des sondes, que les volumes de couplages de celles-ci

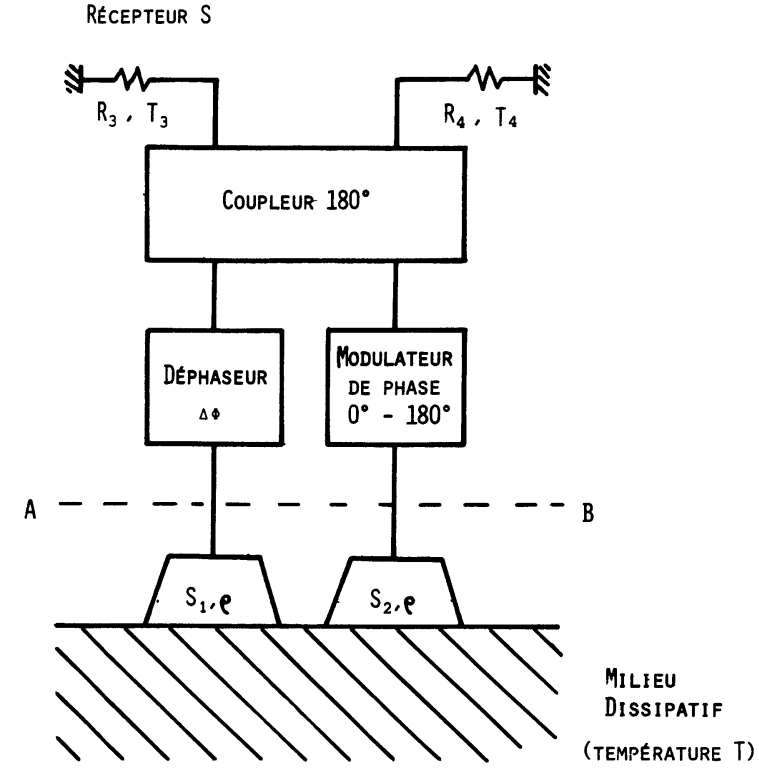

Fig. 7. - Etude du signal détecté en présence d'un milieu dissipatif à température uniforme.

[Synoptic of the experimental set up (we consider the temperature distribution of the lossy material to be uniform.).]

aient une partie commune ou non. En particulier, dans ces conditions, la somme des termes donnés par la relation (8), étendue à la partie commune des volumes de couplage est nulle (milieu dissipatif à température uniforme).

Cette propriété a été vérifiée expérimentalement dans notre laboratoire sur des liquides portés à des températures largement variables.

Cette propriété importante différencie notablement la T.M.O.C. de la T.M.O. classique à une seule sonde. Elle indique que la T.M.O.C. devra être nécessairement utilisée pour la détection de gradients thermiques spatiaux.

Par ailleurs, la propriété qui vient d'être établie indique que l'intégrale de l'expression (8) étendue au volume commun est nulle lorsque $T_{i}$ est constant

$$
\sum C_{i 1} C_{i 2} \cos \Psi \cos \left(\Phi_{i 1}-\Phi_{i 2}\right)=0 .
$$

Les grandeurs $C_{i}, \Phi_{i}$ et $\Psi$ qui caractérisent le couplage entre les volumes élémentaires et les sondes correspondent aux champs rayonnés en mode actif [12] (amplitude, phase, orientation). Ces grandeurs sont obtenues soit par le calcul, soit expérimentalement en déplaçant une petite antenne dans le milieu dissipatif.

L'expression (19) fournit un moyen original de vérifier la validité de l'ensemble de ces données $C_{i}$, $\Phi_{i}$ et $\Psi$ : d'après ce test les données utilisées sont d'autant plus exactes que la grandeur $\varepsilon$ définie comme suit est plus faible.

$$
\varepsilon=\frac{\sum C_{i 1} C_{i 2} \cdot \cos \Psi \cdot \cos \left(\Phi_{i 1}-\Phi_{i 2}\right)}{\sum C_{i}^{2}} .
$$


Le terme figurant au dénominateur, correspond à une intégration effectuée sur le volume couplé à une seule sonde ; il permet de normaliser le paramètre $\varepsilon$.

Notons par ailleurs que l'on devrait aussi envisager l'influence du couplage entre les sondes $S_{1}$ et $S_{2}$ : dans ces conditions les signaux émis par une même source (soit $R_{3}$ soit $R_{4}$ ) pourraient interférer après avoir transité respectivement soit par $S_{1}$ puis $S_{2}$, soit par $S_{2}$ puis $S_{1}$. Dans les conditions expérimentales où nous travaillons actuellement, cet effet peut être négligé : le couplage que nous avons mesuré entre $S_{1}$ et $S_{2}$ est toujours inférieur à -20 ou $-30 \mathrm{~dB}$.

4.2 LES SONDES NE SONT PAS PARFAITEMENT ADAPTÉES AU MILIEU DISSIPATIF. - La propriété établie précédemment selon laquelle un milieu dissipatif de température uniforme n'a aucune contribution sur le signal de sortie continue d'être applicable. En outre, les signaux thermiques émis par les charges $R_{3}$ et $R_{4}$ peuvent contribuer au signal capté par le récepteur compte tenu d'effets de réflexion au niveau de l'ouverture des sondes. Un raisonnement du type de celui présenté en paragraphe 3 montre que lorsque le modulateur est en position $0^{\circ}$ les signaux reçus par le récepteur issus de $R_{3}$ et $R_{4}$ ont pour expression

$$
v^{\prime}=\frac{\rho}{2}\left[V _ { 3 } \left\lfloor\Delta \Phi+V_{3} \underline{\llcorner}^{\circ}+V_{4}\left\llcorner\Phi+V_{4}\lfloor[] .\right.\right.\right.
$$

Lorsque le modulateur est en position $180^{\circ}$, on a

$$
v^{\prime \prime}=\frac{\rho}{2}\left[V_{3} \Delta \Phi+V_{3}\left\lfloor\Pi+V_{4}\left\lfloor\Delta \Phi+V_{4}\lfloor\underline{0}]\right.\right.\right.
$$

avec

$$
\begin{aligned}
& v_{3}(t)=V_{3} \underline{\llcorner}^{\circ} \\
& v_{4}(t)=V_{4} \underline{L}^{\circ}
\end{aligned}
$$

les signaux de bruit émis par $R_{3}$ et $R_{4}$ portées aux températures $T_{3}$ et $T_{4} . \rho$ est le coefficient de réflexion à l'interface, considéré identique pour les deux sondes.

Compte tenu du théorème de Nyquist, le signal moyen détecté dû à ces contributions vaut

$$
P_{\mathrm{s}}^{\prime}=k \frac{\left|\rho^{2}\right|}{2}\left(T_{3}-T_{4}\right) \cos \frac{2 \Pi \Delta l}{\lambda} .
$$

Si ce terme s'avérait gênant pour certaines applications pratiques - qui visent surtout à étudier la température du milieu dissipatif - on pourra facilement l'annuler en portant les charges $R_{3}$ et $\dot{R}_{4}$ à la même température.

\section{Contribution au signal de sortie du bruit émis par un volume élémentaire situé dans un plan de symétrie de la sonde.}

L'étude que nous développons actuellement a pour but d'évaluer les nouvelles possibilités que l'on peut espérer tirer de la TMOC pour les méthodes de diagnostic médical par exemple pour une meilleure détermination de gradients thermiques régnant dans

les tissus vivants. Pour celà, nous étudions d'abord la contribution au signal de sortie d'un volume élémentaire faisant partie du milieu dissipatif. On considère ici le cas d'un milieu dissipatif propice aux expériences de laboratoires, l'eau, dont les propriétés diélectriques ne sont pas très éloignées de celles de certains tissus (muscle, tissus cérébraux...). On peut alors étudier l'influence de plusieurs paramètres

- la disposition des sondes; jointes selon leur grand ou leur petit côté ; ayant une arête commune, et faisant entre elles un angle $\theta$ (Figs. : 8A, 8B, 8C, 8D);

- la position du volume élémentaire dans le liquide (Fig. 8E);

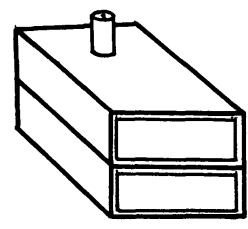

(8 A)

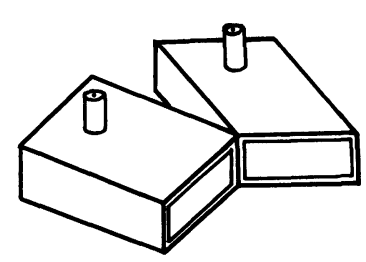

(8 c)

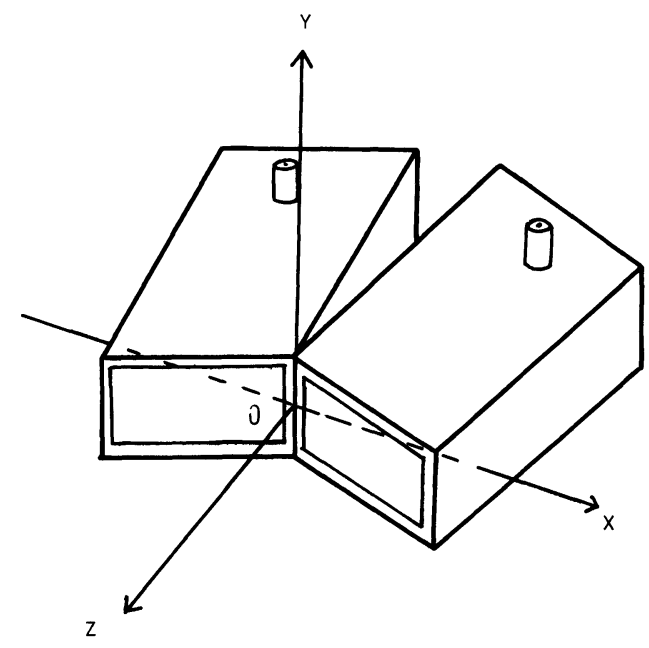

$(8 E)$

Fig. 8. - A, B, C, D : Disposition relative des sondes. Caractéristiques des sondes : ouverture $21,6 \times 10,8 \mathrm{~mm}$, $\varepsilon_{1}=25$. $\mathrm{E}:$ Définition de la position du volume élémentaire dans le trièdre oxyz.

[A, B, C, D : Relative positions of the probes. Characteristics of the probes : aperture $21.6 \times 10.8 \mathrm{~mm}, \varepsilon_{1}=25 . \mathrm{E}$ : Definition of the location of a subvolume $\Delta V$ by its coordinates $x, y$ and $z$.] 
- l'introduction dans le corrélateur d'une différence de marche $\Delta l$ variable (Fig. 4) conduisant à une modulation de l'écart de phase $\Delta \Phi$.

Ces études sont menées :

- soit en simulant l'émission thermique à l'aide d'une diode de bruit (diode avalanche) de petite dimension, plongée dans le liquide. Nous avons vérifié que l'onde émise est alors polarisée, et que le diagramme de rayonnement dans le plan perpendiculaire à l'axe de la diode est isotrope ;

- soit en travaillant en régime monochromatique en émettant un signal à l'aide d'une petite antenne placée dans le liquide;

- soit en calculant les termes de la relation (8) à partir des paramètres $C_{i}, \Phi_{i}$ et $\Psi$ déduits de la connaissance des champs électriques rayonnés en mode actif.

Les figures 9 à 11 présentent des résultats de ce type obtenus avec une diode avalanche.

La figure 9 donne la réponse obtenue lorsque la source de bruit, placée à une certaine profondeur $\left(z_{1}=2-3-4 \mathrm{~cm}\right)$, est déplacée parallèlement à la surface du liquide. Dans ce cas, les sondes sont jointes selon leur petit côté. (On considère ici que $y=0$.)

Ce type de résultat indique déjà la nature profondément différente des données fournies par la thermographie microonde classique (à une seule sonde) et la T.M.O. par corrélation; en particulier, pour certaines positions de l'objet on obtient une contribution négative au signal de sortie. Notons que compte tenu du fait que la méthode est insensible à l'émission d'un milieu porté à une température uniforme et des conditions de l'expérience, l'aire sous les courbes $S\left(x_{1}\right)$ à $z_{1}=$ Cte doit être nulle. Notons aussi que la position $x_{1}$ des zéros dépend de la profondeur

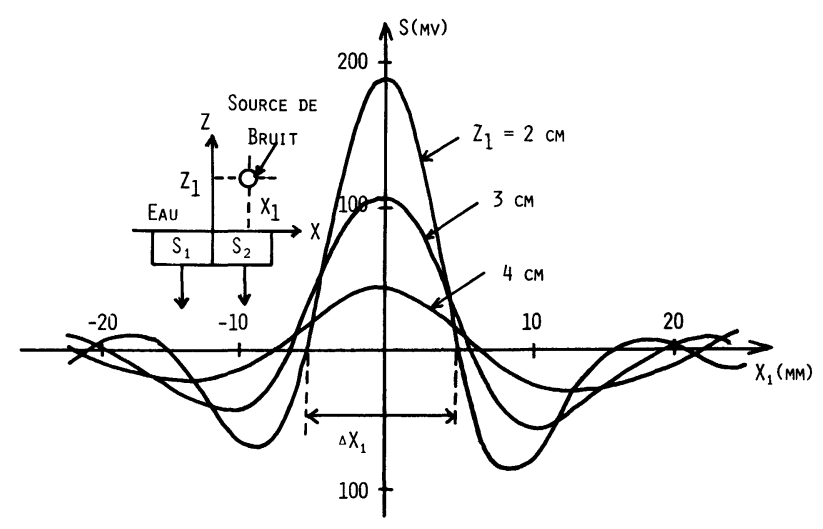

Fig. 9. - Réponse du thermographe à corrélation (bande $\mathrm{S}$ ) lorsqu'une source de bruit située dans l'eau à plusieurs profondeurs $(2-3-4 \mathrm{~cm})$ est déplacée parallèlement à la surface. Les sondes sont parallèles, jointes sur leur petit côté.

[Experimental output signal obtained by Correlation Microwave Thermography for a noise source submerged in water $(\theta=0, \Delta l=0)$. The probes are parallel, joined on their small side.] de l'objet; ceci est aussi une propriété nouvelle que nous allons exploiter dans la méthode décrite au paragraphe 6.

La figure 10 donne une réponse du même type, pour $z_{1}=1,2 \mathrm{~cm}$ et pour différentes valeurs de l'angle $\theta$ entre les sondes (celles-ci sont jointes selon le petit côté de l'ouverture). On constate une modification importante des réponses en fonction de $\theta$, et en particulier une évolution rapide du signal fonction de $x_{1}$ lorsque $\theta$ èst le plus important.

La figure 11 traite le cas de la source de bruit déplacée sur l'axe du système. Plusieurs valeurs de $\theta$ sont considérées. On constate encore une différente très nette par rapport aux données de la Thermographie Microonde à une sonde : la T.M.O.C. est moins sensible à l'émission proche et - dans le cas considéré - privilégie les sources situées à une distance comprise entre 1 et $2 \mathrm{~cm}$.

\section{Détermination de la profondeur d'une source ponc-} tuelle par action sur la différence de marche $\Delta l$ introduite dans le corrélateur.

On peut modifier les réponses telles celles présentées figures 9,10 et 11 en introduisant une différence de marche $\Delta l$ dans l'un des bras du corrélateur, soit un déphasage $\Delta \Phi=2 \Pi \Delta l / \lambda$ si l'on considère un fonctionnement monochromatique. Les diagrammes donnés figure 12 concernent une source monochromatique (antenne miniature alimentée à $3 \mathrm{GHz}$ ) placée dans l'eau à une profondeur $z_{1}=2 \mathrm{~cm}$; on a enregistré les signaux détectés fonction de $x_{1}$ pour des valeurs de $\Delta l$

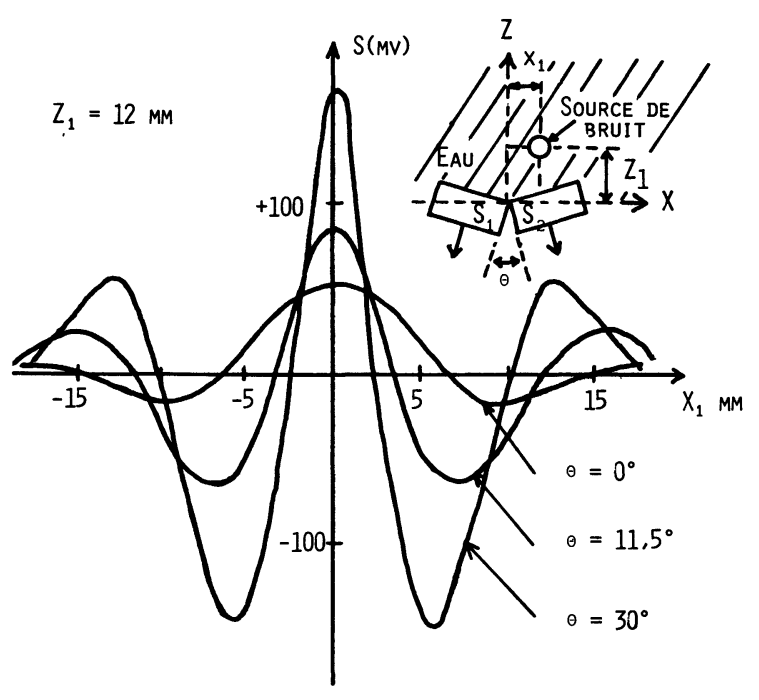

Fig. 10. - Réponses du thermographe à corrélation bande $\mathrm{S}$ lorsqu'une source de bruit située dans l'eau à la profondeur $z_{1}=12 \mathrm{~mm}$, est déplacée parallèlement à la surface. Les sondes, appliquées selon une arête de l'ouverture (petit côté) font entre elles un angle $\theta\left(0^{\circ}, 11^{\circ} 5,30^{\circ}\right)$.

[Output signal $S$ (experiment) obtained by Correlation Microwave Thermography for a noise source submerged in water for different values of $\theta(\Delta l=0)$. The probes are parallel, joined on their small side.] 


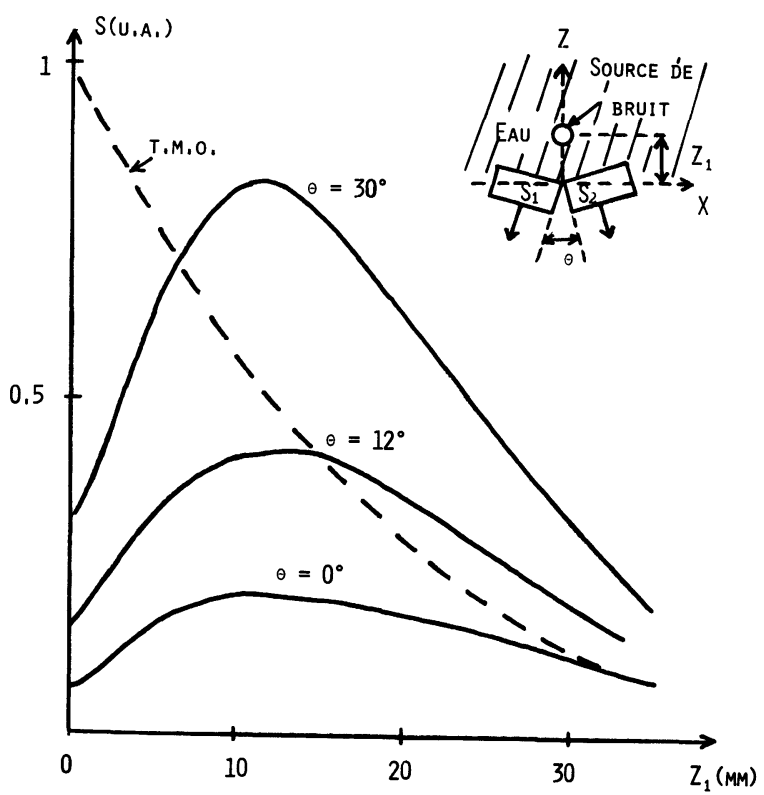

Fig. 11. - Réponse du thermographe à corrélation bande $\mathrm{S}$ lorsque la source de bruit située dans l'eau, est déplacée sur l'axe du système. Plusieurs valeurs de $\theta$ sont envisagées. Ces réponses sont comparées à celle de la thermographie à une seule sonde (courbe en pointillés).

[Output signal $S$ (experiment) for a noise source submerged in water. Comparison of the data obtained by MWT (---) and CMWT $(\longrightarrow)$ for $\theta=0,12^{\circ}$ and $30^{\circ}$.]

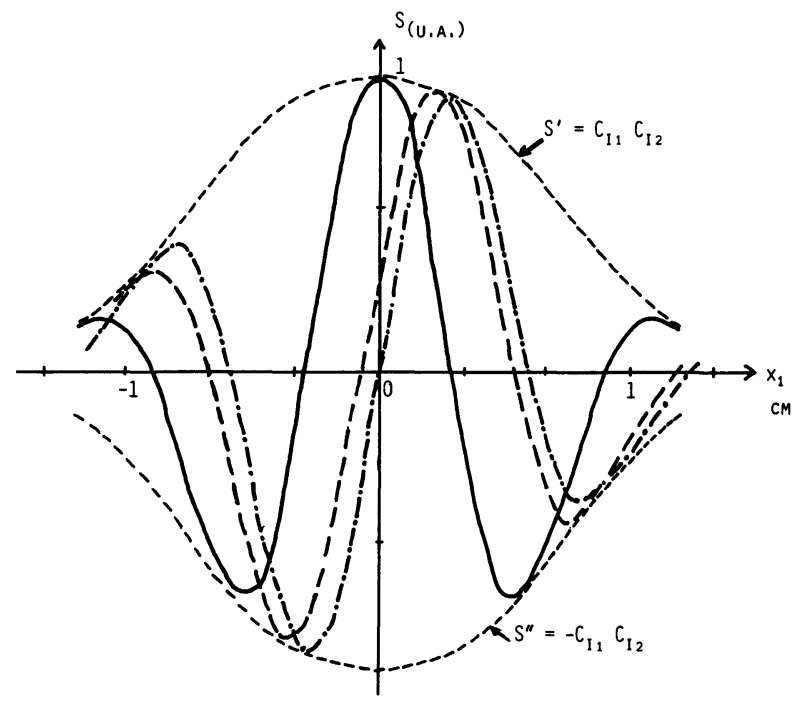

Fig. 12. - Réponse du thermographe à corrélation à une antenne miniature, plongée dans l'eau, alimentée à $3 \mathrm{GHz}$, (profondeur $2 \mathrm{~cm}$ ) déplacée parallèlement à la surface. (Sondes parallèles jointes sur leur petit côté.) $-\Delta l=0$, $----\Delta l=2 \mathrm{~cm},-.-.-\Delta l=2,5 \mathrm{~cm}$.

[Output signal of the CMWT when a small antenna submerged in water fed with a signal at $3 \mathrm{GHz}$, is moved parallel to the surface (depth $2 \mathrm{~cm}$; probes parallel joined on their small side $; \theta=0)$. $-\Delta l=0,---\Delta l=2 \mathrm{~cm},-.-.-.-$ $\Delta l=2.5 \mathrm{~cm}$.] égales à 0,2 et $2,5 \mathrm{~cm}$. Remarquons que ces diagrammes sont en accord avec la relation (8) et admettent pour enveloppes les courbes $S^{\prime}=C_{i 1} C_{i 2}$ et $S^{\prime \prime}=-C_{i 1} C_{i 2}$; la différence de marche $\Delta l$ qui annule le signal détecté $\left(\Delta l_{(S=0)}\right)$ dépend à la fois de $z_{1}$ et de $x_{1}$. On peut exploiter cette propriété pour déterminer la profondeur $z$ d'une source. Nous proposons l'utilisation de l'abaque donné figure 13 qui représente $\Delta l_{(S=0)}$ fonction de $z_{1}$ pour différentes valeurs de $x_{1}$. Ce mode opératoire est le suivant.

On réalise d'abord $\Delta l=0$ et on déplace les sondes à la surface du liquide jusqu'à l'obtention d'un signal maximal; on définit ainsi la position $x_{1}=0$. On déplace alors les sondes d'une distance $x_{1}$ connue, et l'on recherche l'annulation du signal de sortie par action sur $\Delta l$. Le rapport de la valeur correspondante de $\Delta l_{(S=0)}$ sur l'abaque (Fig. 13) conduit à la détermination de la profondeur $z_{1}$ de la source. Notons que cette méthode est intéressante car - dans la mesure où elle peut s'appliquer - elle permet de déterminer $z_{1}$ en travaillant à une seule fréquence alors que la détermination de la profondeur par les méthodes de T.M.O. classiques nécessitent d'opérer au moins à deux fréquences [16]. Remarquons aussi que ce procédé est fondé sur un fait déjà observé sur la figure 11,

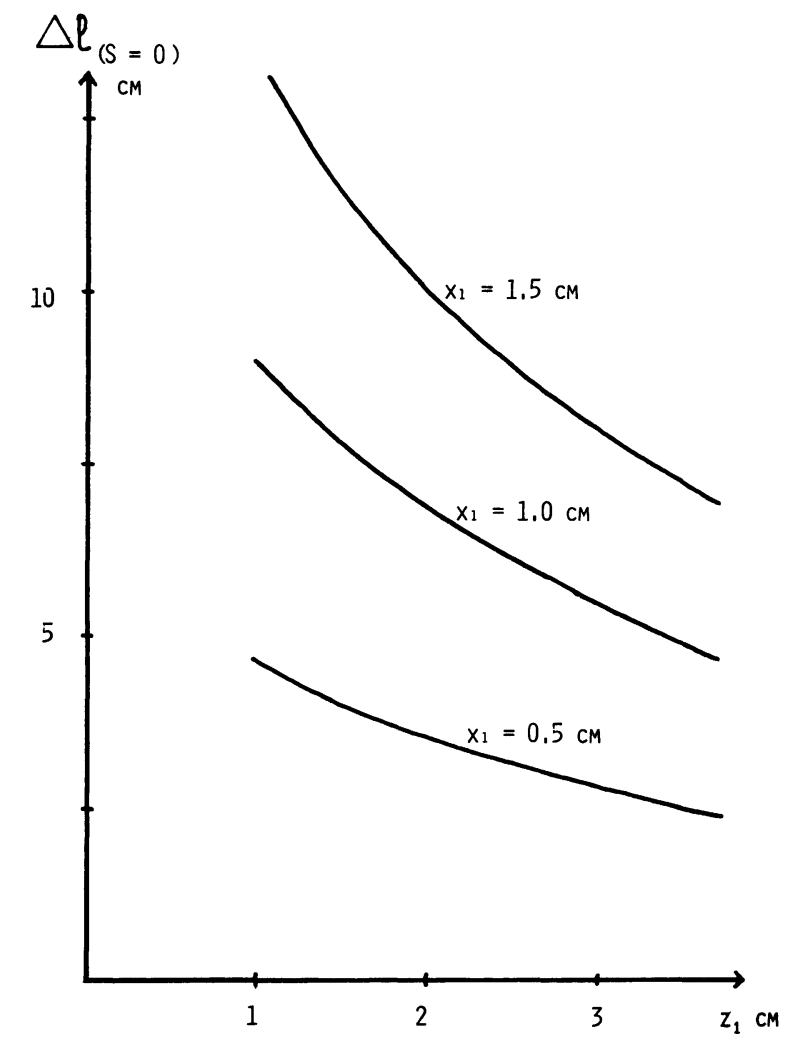

Fig. 13. - Abaque permettant de déterminer la profondeur d'une source ponctuelle immergée dans l'eau à partir de connaissance de la différence de marche $\Delta l(S=0)$ annulant le signal de sortie (fréquence $3 \mathrm{GHz}$ ).

[Diagram used in order to determine the depth of a point source submerged in water : this method needs the knowledge of $\Delta l$ for which the output signal $S$ is zero (frequency $3 \mathrm{GHz}$ ). ] 
qui indique déjà que la valeur de $x_{1}$ produisant une annulation du signal dépend de la profondeur de la source. En fait, annuler $S$ consiste (relation (8)) à réaliser ( $N$ étant un entier).

$$
\Phi_{i 1}-\Phi_{i 2}+2 \Pi \frac{\Delta l}{\lambda}=\frac{\Pi}{2}+2 N \Pi .
$$

Ce but peut être atteint de plusieurs façons : soit en modifiant la quantité $\left(\Phi_{i 1}-\Phi_{i 2}\right)$ par action sur $x_{1}$; soit en modifiant $\Delta \Phi$ par action sur $\Delta l$. Compte tenu du fait que les phases $\Phi_{i 1}$ et $\Phi_{i 2}$ concernent une propagation dans un milieu de forte permittivité et que une modification de $\Delta \Phi$ concerne une propagation dans l'air, cette action sur $\Delta l$ met en jeu des dimensions géométriques plus importantes que dans le premier cas. Par conséquent, la deuxième solution conduit à une commande beaucoup plus sensible du signal de sortie.

\section{Etudes sur modèles. Sensibilité de la méthode.}

Les études telles celles décrites en 5 et 6 concernent la contribution d'un volume élémentaire au signal de sortie; elles nous ont permis de bien comprendre les mécanismes propres à la T.M.O.C. et de mettre au point un premier prototype.

Parallèlement sónt conduites des études sur des modèles constitués par des objets thermiques : on relève par exemple le signal obtenu par T.M.O.C. lorsque les sondes sont déplacées sur le fond d'une cuve remplie d'eau. Dans le cas envisagé figure 14, un cylindre rempli d'eau tiède est placé dans la cuve; dans le cas de la figure 15 , un plan perpendiculaire à la surface sépare deux volumes à des températures différentes. Des comparaisons entre techniques T.M.O. et T.M.O.C. sont effectuées sur ces modèles, donnant souvent l'avantage à la T.M.O.C. en ce qui concerne la résolution spatiale.

Des expériences de ce type sont poursuivies actuellement et nous effectuons des calculs, par application de la relation (8), à partir des expressions des paramètres définissant le couplage des volumes élémentaires déduits d'expérience effectuées en mode actif.

Parallèlement nous menons des études qui ont pour but la comparaison de la sensibilité en T.M.O. et en T.M.O.C., ces résultats seront présentés ultérieurement.

\section{Conclusion.}

Nous avons défini une nouvelle méthode de radiométrie fondée sur la détection cohérente du bruit thermique. Un système de T.M.O.C. fonctionnant en bande $\mathrm{S}$ a été mis au point, et a servi à effectuer un certain nombre d'essais préliminaires qui nous ont permis de comprendre les caractéristiques de cette nouvelle technique et, après comparaison avec la T.M.O. classique, à prévoir un gain de résolution spatiale dans la localisation des gradients thermiques.

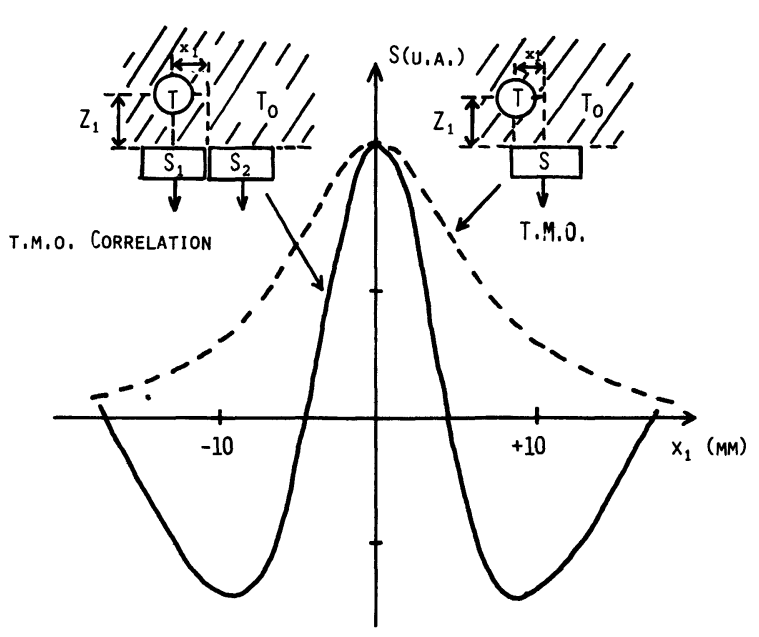

Fig. 14. - Comparaison des signatures thermiques obtenues par T.M.O. et par T.M.O.C. Cas d'un cylindre immergé dans le même milieu dissipatif à température ambiante (eau).

[Experiments on a thermal structure (cylinder in water; $D=1.1 \mathrm{~cm}, z_{1}=15 \mathrm{~mm}$ ) showing a more selective output signal by CMWT.]

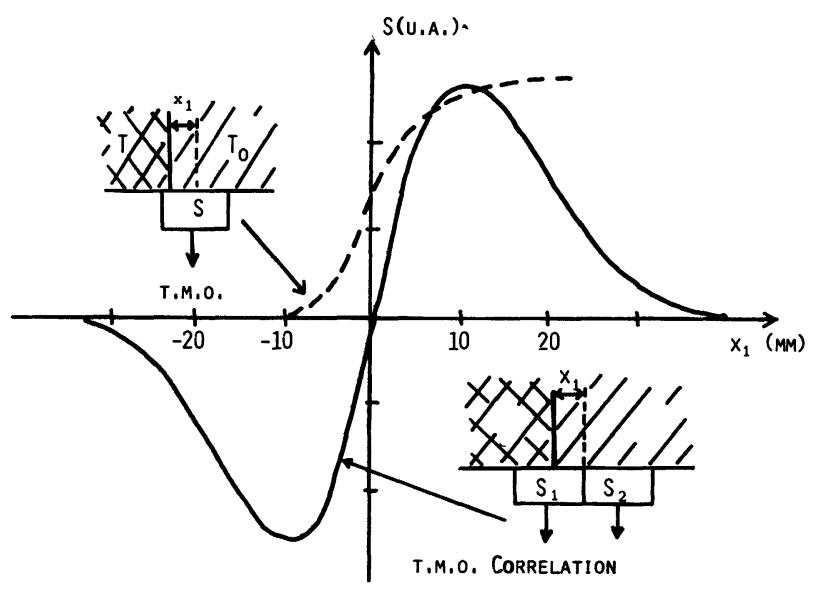

Fig. 15. - Comparaison des signatures thermiques obtenues par T.M.O. et par T.M.O.C. Cas d'un milieu dissipatif homogène (eau) dont deux parties - séparées par un plan perpendiculaire à la surface - sont à des températures différentes.

[Experiments in water with two compartments at different temperatures (MWT and CMWT).]

Cette étude se poursuit maintenant dans le but de coupler ces deux méthodes de Thermographie Microonde et d'essayer de les utiliser conjointement dans le domaine des applications médicales.

\section{Remerciements.}

Les auteurs remercient la D.G.R.S.T. (T.L.B.M. Hyperthermie), l'ANVAR, la Région Nord Pas de Calais pour leur aide financière. 
Annexe 1.

Nous expliquons ici la présence du terme $\cos \Psi$ dans les relations (6), (7) et (8) exprimant la contribution au signal de sortie du corrélateur d'un volume élémentaire $\Delta V_{i}$ couplé aux deux sondes. $\Psi$ est l'angle que font les directions des champs électriques rayonnés (en mode actif) par les deux sondes au point considéré.

L'émission thermique d'un milieu dissipatif résulte de l'addition des rayonnements individuels incohérents des molécules ou groupes de molécules qui le constituent. Les signaux émis par ces différentes sources sont aléatoires et ne présentent aucune relation de phase entre eux. Du point de vue électromagnétique, le milieu est assimilable à un ensemble de dipôles élémentaires ayant des orientations aléatoires pouvant varier au cours du temps et dont la distribution angulaire peut être supposée uniforme.

Un volume élémentaire $\Delta V_{i}$ est constitué d'un grand nombre de ces émetteurs. Estimons, à un instant donné, sa contribution au signal de sortie (relations (6) et (7)).

Appelons $\mathbf{E}_{1}$ et $\mathbf{E}_{2}$ les directions des champs rayonnés en mode actif en $\Delta V_{i}$ par les sondes $S_{1}$ et $S_{2}$. Chaque émetteur va contribuer aux termes $T_{i} C_{i 1}^{2}$ et $T_{i} C_{i 2}^{2}$ compte tenu des composantes du champ qu'il émet selon les directions $\mathbf{E}_{1}$ et $\mathbf{E}_{2}$. Ces termes ne dépendent pas des orientations de $\mathbf{E}_{1}$ et $\mathbf{E}_{2}$ puisque la distribution angulaire des émetteurs est uniforme.

Il n'en est pas de même des termes croisés, ou corrélés, obtenus après la détection quadratique. Considérons un émetteur rayonnant un champ donné. Les composantes de ce champ, qui sont perpendiculaires au plan défini par $\mathbf{E}_{1}$ et $\mathbf{E}_{2}$ sont inactives. Il suffit donc de raisonner dans le plan $E_{1} E_{2}$. La contribution d'un tel champ $E$ (Fig. 16) au terme croisé du signal de sortie est du type

$$
C_{i 1} \cdot \cos \left(\gamma-\gamma_{1}\right) \cdot C_{i 2} \cdot \cos \left(\gamma-\gamma_{2}\right) \cos \left(\Phi_{i}-\Phi_{i 2}-\Delta \Phi\right)
$$

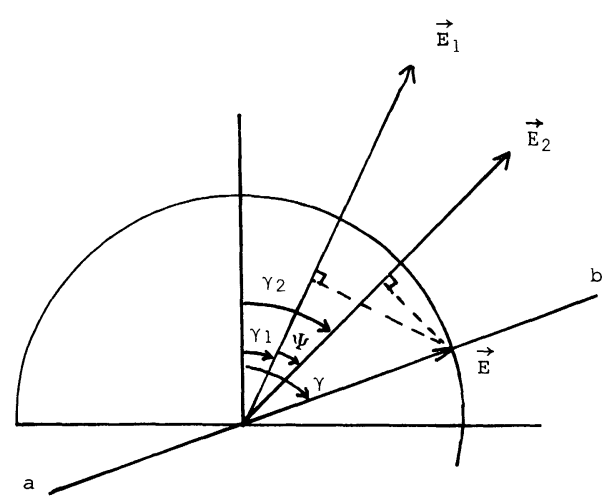

Fig. 16. - Situation d'un dipôle rayonnant élémentaire (E) par rapport aux champs $\mathbf{E}_{1}$ et $\mathbf{E}_{2}$ rayonnés en mode actif par les deux sondes.

[Situation of a radiating elementary dipole $(\mathbf{E})$ with respect to the fields $\mathbf{E}_{1}$ and $\mathbf{E}_{2}$ radiated in active process by the two probes.]

avec $\gamma, \gamma_{1}$ et $\gamma_{2}$ les angles définissant les orientations de $E, E_{1}$ et $E_{2}$.

En fait, à un instant donné le champ $\mathbf{E}$ est dirigé, soit de $a$ vers $b$, soit de $b$ vers $a$. Dans les deux cas, sa contribution au signal de sortie est donné par la relation (27). L'estimation du signal croisé total fourni par $\Delta V_{i}$ revient à intégrer la contribution des champs $E$ pour des angles $\gamma$ compris entre $-\Pi / 2$ et $+\Pi / 2$.

Comme la distribution angulaire des champs rayonnés $E$ est uniforme et que

$$
\int_{-\Pi / 2}^{+\Pi / 2} \cos \left(\gamma-\gamma_{1}\right) \cos \left(\gamma-\gamma_{1}\right) d \gamma=2 \cos \Psi
$$

on en déduit immédiatement les relations (6), (7) et (8).

\section{Bibliographie}

[1] Gautherie, M., Moyses, B., Leroy, Y., Mamouni, A., Moschetto, Y., SozANSKI, J. P., Imagerie thermique au moyen de systèmes radiométriques microondes appliquée à la détection et au pronostic de tumeurs mammaires, cérébrales et thyroïdiennes, Symposium International Ondes Electromagnétiques et Biologie,' Jouy-en-Josas, 4 juillet 1980.

[2] Thouvenot, P., Robert, J., Mamouni, A., Renard, C., Microwave Thermometry in intracranial Pathology, Biomedical Thermology (Alan R. Lyss, Inc. 150 Fifth Avenue, New York, NY 10011) 1982, p. 501-508.

[3] Besson, P., Chive, M., Leroy, Y., Thermographie
Microonde à $3 \mathrm{GHz}$ Application à l'exploration du sein. NPN Médecine, II nº 28 (1982).

[4] Mamouni, A., Radiométrie Hyperfréquence. Application à la mesure atraumatique de la température au sein des tissus vivants (thermographie microonde). Thèse de $3^{\mathrm{e}}$ cycle, Lille, 20 novembre 1978.

[5] NGUYEN, D. D., Thermographie et chauffage microonde. Contribution à la conception et à la réalisation de systèmes destinés au Génie Biologique et Médical. Thèse de 3e cycle, Lille, 10 décembre 1980.

[6] LEROY, Y., Microwave Radiometry and Thermography. Present and Prospective. Biomedical Thermology. (Publ. Alan Lyss N.Y.) 1982, p. 485-499. 
[7] LEROY, Y., Microwave thermography for medical applications. Conférence Invitée Workshop on medical applications of microwave. 12th European Microwave Conference, 13-17 septembre 1982, Helsinki (Finlande).

[8] Audet, J., Bolomey, J. Ch., Pichot, C., Nguyen, D. D., Robillard, M., ChIVE, M., Leroy, Y., Electrical characteristics of waveguide applicators for medical applications. J. Microwave Power, $15 \mathrm{n}^{\circ} 3$ (1980).

[9] Giaux, G., Prevost, B., Leroy, Y., Chive, M., PlanCOT, M., Local Hyperthermia of Cancers, by Radiofrequency or Microwaves, in combination with radiotherapy or chemotherapy, controlled by microwave thermography : technical aspects; first clinical observations. 4th Eur. Hyperth. Meeting, London, 1-2 juillet 1982.

[10] Chive, M., Plancot, M., Leroy, Y., Giaux, G., Prévost, B., Microwave ( $1 \mathrm{GHz}$ and $2.45 \mathrm{GHz}$ ) and radiofrequency (13.56 MHz) Hyperthermia monitored by microwave thermography. European Microwave Conference; 13-17 septembre 1982, Helsinki (Finlande).

[11] Dicke, R. H., The measurement of thermal Radiation at Microwave Frequencies, Rev. Sci. Instrum. 17 $\mathrm{n}^{\mathrm{o}} 7$ (1946) 228-275.

[12] Robillard, M., Contribution à l'étude des sondes et à la Reconnaissance d'Objet Thermique par Thermographie Microonde (T.M.O.). Thèse de 3 e cycle, Lille, 12 novembre 1981.
[13] FABRE, J. J., Méthodes de calcul de signaux thermiques et possibilité de nouvelles utilisations de la thermographie microonde. Thèse de $3 \mathrm{e}$ cycle, Lille, 22 septembre 1982.

[14] Robillard, M., Chive, M., Leroy, Y., Toward an interpretation of the thermal signatures achieved by microwave thermography. Microwave Power Symposium, June 1981, Toronto (Canada).

[15] Robillard, M., Chive, M., Leroy, Y., Audet, J., Pichot, Ch., Bolomey, J. Ch., Microwave thermography, Characteristics of waveguide applicators and signatures of thermal structures, J. Microwave Power $17 \mathrm{n}^{\circ} 2$ (1982).

[16] Hochedez-Robillard, M., Leroy, Y., à paraître.

[17] RyLE, M., A new radio interferometer and its application to the observation of weak radio stars, Proc. R: Doc. London 211 (1951) 351-374.

[18] Mamouni, A., Van de Velde, J. C., Leroy, Y., New Correlation Radiometer for Microwave Thermography, Electron. Lett. $17 \mathrm{n}^{\mathrm{o}} 16$ (1981) 554-555.

[19] BellarBI, L., Etudes préliminaires sur la Thermographie Microonde par Correlation, Diplôme d'Etudes Approfondies, Univ. de Lille I, 1982.

[20] Mamouni, A., Leroy, Y., Van de Velde, J., Bellarbi, L., Introduction to correlation microwave thermography. 12th European Microwave Conference, 13-17 septembre 1982, Helsinki (Finlande). 